\title{
BMJ Open Determinants of treatment duration in the prevention of recurrent venous thromboembolism: a protocol for a balanced vignette experiment
}

\author{
Vincent Ten Cate, ${ }^{1,2}$ Brigitte AB Essers, ${ }^{3}$ Martin H Prins ${ }^{1}$
}

To cite: Ten Cate V, Essers BAB, Prins MH. Determinants of treatment duration in the prevention of recurrent venous thromboembolism: a protocol for a balanced vignette experiment. BMJ Open 2017;7:e015231. doi:10.1136/ bmjopen-2016-015231

- Prepublication history for this paper is available online. To view please visit the journal online (http://dx.doi.org/ 10.1136/ bmjopen-2016-015231).

Received 18 November 2016 Revised 9 March 2017 Accepted 17 March 2017

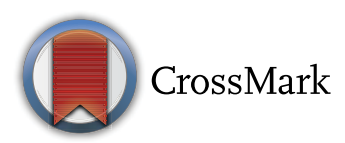

${ }^{1}$ Department of Epidemiology, Maastricht University Medical Center, Maastricht, The Netherlands

${ }^{2}$ Maastricht University CAPHRI School for Public Health and

Primary Care, Maastricht, The Netherlands

${ }^{3}$ Department of Clinical Epidemiology and Medical Technology Assessment, Maastricht University Medical Center, Maastricht, The Netherlands

Correspondence to Mr Vincent Ten Cate; vincent.ten.cate@mumc.nl

\section{ABSTRACT}

Introduction Venous thromboembolism (VTE) is a condition that annually occurs in approximately $1 \%$ of the world's population. Patients who have already had a VTE are at elevated risk for a recurrent VTE. Recurrent events increase the risk of long-term sequelae and can be fatal. Adequate secondary prophylaxis is thus needed to prevent such events. Patients with VTE are often prone to bleeding, and pharmacological prophylaxis exacerbates bleeding risk. Expert opinions on the optimum duration of secondary prophylaxis in VTE still vary substantially. The existence of treatment guidelines has not led to uniformity of VTE secondary prophylaxis strategies, which means that physicians still adhere to individual risk calculi in determining treatment duration.

Methods and analysis The aim of this study is to establish what factors lie at the root of this variance in VTE secondary prophylactic treatment strategies, and what risk factors are deemed of particular importance in determining the perceived risks and benefits of variable treatment durations. To do this, we created a survey based on a D-efficient and G-efficient balanced experimental vignette design. This protocol covers all aspects of how this survey was set up and how it was implemented. The analysis of the experimental data will be carried out using mixedeffects methods, which are beneficial in scenarios with high interindividual variance and correlated (eg, repeatedmeasures) responses. We propose the use of maximal random effects structures insofar as possible.

Ethics and dissemination All data are de-identified, and any identifying characteristics of the respondents will not be reported in a final manuscript or elsewhere. A paper describing the expert interviews is currently under peer review. A manuscript that contains the analysis of the results of the experiment described in this protocol is being drafted, and will also be submitted to a peerreviewed journal.

\section{INTRODUCTION}

Venous thromboembolism (VTE) is a condition that annually occurs in approximately $1 \%$ of the world's population. ${ }^{1}$ VTE encompasses deep vein thrombosis (DVT) and pulmonary embolism (PE). The elderly are at particular risk, as the incidence of VTE increases with age to approximately $5 \% 0-6 \%$ o
Strengths and limitations of this study

The experiment uses a D-efficient and G-efficient balanced fractional factorial design.

- The design was inspired by and modified based on interactions with several specialists in the field.

- Mixed effects methods are used to interpret and account for interindividual variance.

- Not all relevant risk factors were incorporated in the model due to technical constraints.

- Due to the nature of the experiment, the risk factors featured in it do not necessarily occur in real-life distributions.

in those over 80 -years-old. ${ }^{1}$ Two-thirds of VTE diagnoses constitute cases of deep vein thrombosis, and the remainder pulmonary embolism. $25 \%-50 \%$ of VTE cases are idiopathic, which means that they are of unknown aetiology. ${ }^{2}$

Approximately $6 \%$ of DVT cases and $12 \%$ of PE cases are lethal within one month of diagnosis. ${ }^{2}$ Recovery rates from a VTE event are high given appropriate pharmacological anticoagulant therapy. ${ }^{2}$ If the embolus in patients with PE is not completely resolved after treatment, long-term complications such as chronic thromboembolic pulmonary hypertension (CTPH) may occur. ${ }^{3}$ Similarly, patients with DVT are at elevated risk of post-thrombotic syndrome (PTS) during long-term follow-up. Moreover, patients who have already had a VTE are at elevated risk for a recurrent VTE. ${ }^{4}$ Recurrent events increase the risk of long-term sequelae and are potentially fatal. ${ }^{4}$ Hence, adequate secondary prophylactic treatment, that is, the outpatient preventive treatment that follows the resolution of an acute event, is necessary. However, what exactly constitutes adequate secondary prophylaxis of VTE is still controversial: physicians have yet to achieve consensus on both the ideal type and duration of treatment. ${ }^{5-10}$ 
The difficulty in achieving consensus regarding the duration of secondary thromboprophylaxis in patients with VTE stems from the balance that has to be struck between the thrombotic risk associated with non-treatment, and the reduction in risk of recurrence and the induced bleeding risk associated with anticoagulant therapy. As stated, VTE is a disease that disproportionately affects the elderly, and the elderly are a group particularly prone to bleeding on account of their frailty. Physicians are thus faced with the quandary of prescribing a treatment that itself poses a risk to the health of their patients, while not doing so might incur an even greater risk due to recurrent disease. Their task is then to find the optimum duration of treatment that most effectively mitigates the risk of VTE recurrence in a patient, while minimising their exposure to an inherently risky treatment.

Organisations such as the American College of Chest Physicians (CHEST, formerly: ACCP) and the European Society of Cardiology (ESC) have attempted to coordinate and formalise the treatment and prevention of VTE with periodical instalments of treatment guidelines. ${ }^{11} 12$ However, the recommendations outlined in these guidelines change with every new instalment, and it is unclear how many physicians adhere to these guidelines. Moreover, these guidelines represent a combination of evidence-based research and expert opinions, which themselves vary from physician to physician. The ongoing debate on the optimum type and duration of treatment is testament to the existence of many different calculi individual physicians employ in their long-term preventive VTE treatment strategies.

The aim of this study is to establish what factors lie at the root of this variance in VTE secondary prophylactic treatment strategies, and what risk factors are deemed of particular importance in determining the perceived risks and benefits of ceasing or continuing preventive treatment for a particular duration of time. We do this by having physicians evaluate patient vignettes, that is, hypothetical patient profiles, the attributes of which were derived from expert interviews.

\section{METHODS AND ANALYSIS \\ Vignette experiment}

Vignettes are hypothetical scenarios that mimic reallife situations, enabling researchers to evaluate their phenomenon of interest in a controlled experimental setting. ${ }^{13}$ Since there is little noise in a designed experiment, covariates can be assessed in a relatively unbiased manner. ${ }^{13}$ Additionally, the use of vignettes precludes the need to recruit a balanced sample of subjects, as such a sample can simply be simulated. In our case, we aim to establish what drives the decision-making process surrounding secondary prophylaxis of VTE. In a real-life setting, it would be difficult to recruit a diverse enough set of patients to take the various risk factors that may

Table 1 Frequency table of risk factors mentioned by interviewed physicians

\begin{tabular}{|c|c|c|c|}
\hline Factors (k) & Levels & Type of risk factor & Freq. (\%) \\
\hline Idiopathic & no - yes & TR & $13(100)$ \\
\hline VTE type & $\begin{array}{l}\text { distal DVT - proximal DVT - } \\
\text { non-massive PE - massive PE }\end{array}$ & TR & $13(100)$ \\
\hline Thrombophilia & none - acquired - inherited & TR & $11(85)$ \\
\hline History of bleeding & no - yes & $\mathrm{BR}$ & $10(77)$ \\
\hline $\begin{array}{l}\text { On concurrent antithrombotic } \\
\text { medication (eg, aspirin) }\end{array}$ & no - yes & $\mathrm{BR}$ & $9(69)$ \\
\hline Age & $<65->65$ & $\mathrm{BR} / \mathrm{TR}$ & $8(62)$ \\
\hline Renal function & normal - insufficient & $\mathrm{BR}$ & $7(54)$ \\
\hline Liver disease & no - yes & BR & $7(54)$ \\
\hline Unstable or high INR & no - yes & $\mathrm{BR}$ & $7(54)$ \\
\hline History of thrombosis & no - yes & TR & $6(46)$ \\
\hline BMI & $\begin{array}{l}\text { underweight - normal weight - } \\
\text { overweight }\end{array}$ & $\mathrm{BR} / \mathrm{TR}$ & $5(38)$ \\
\hline Uncontrolled hypertension & no - yes & $\mathrm{BR}$ & $5(38)$ \\
\hline Alcohol or drug abuse & no-yes & $\mathrm{BR}$ & $5(38)$ \\
\hline Family history of VTE & no - yes & TR & $4(31)$ \\
\hline Physical activity & sedentary - active & TR/BR & $4(31)$ \\
\hline Thrombocytopenia & no - yes & BR & $3(23)$ \\
\hline Signs of PTS & no - yes & TR & $3(23)$ \\
\hline Sex & female - male & TR & $2(15)$ \\
\hline
\end{tabular}

BMI, body mass index; BR, bleeding risk; INR, internationalnormalised ratio; PTS, post-thrombotic syndrome; TR, thrombotic risk (ie, VTE recurrence risk); VTE, venous thromboembolism. 
$D$ and $G$ efficiencies of a design matrix $\xi_{n \times k}$

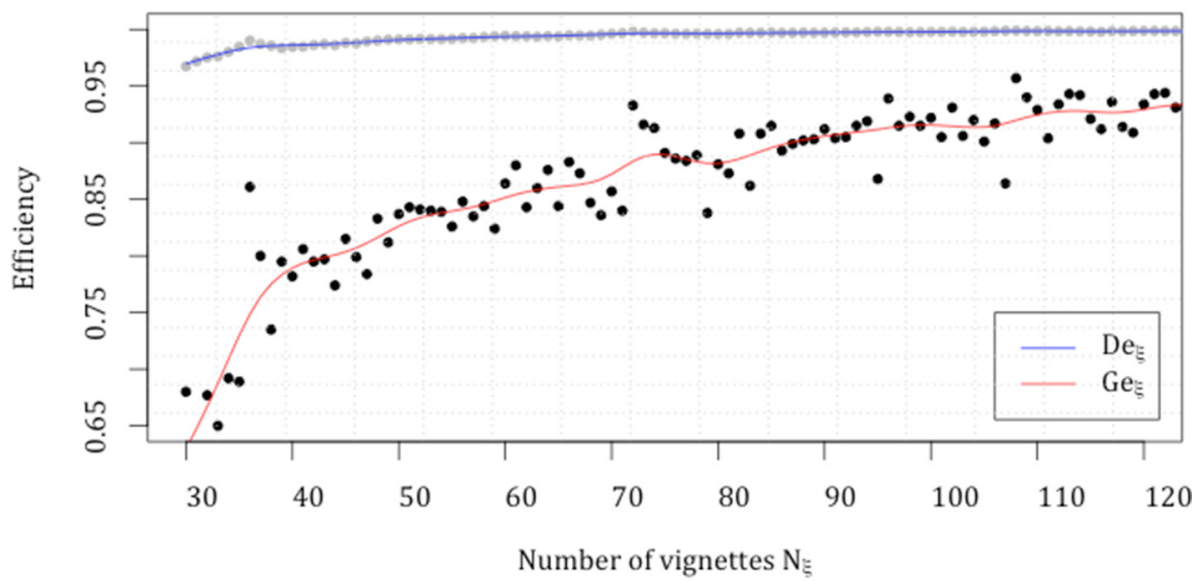

Figure $1 D$ and $G$ efficiencies of design matrix $\xi$.

influence this process into account. With the vignette experiment described in this paper, we achieved a highly efficient alternative to a much more costly and time-consuming real-life experiment.

\section{Preselection of attributes}

From December 2015 to February 2016, we conducted 13 semistructured interviews with an equal number of senior-level physicians affiliated with medical centres around the world that participate in the Einstein Choice study. ${ }^{14}$ The purpose of these interviews was to establish which factors are particularly important in determining the duration of secondary prophylaxis of VTE, and, conversely, which factors weigh against a decision to continue treatment past the resolution of the initial event. Our focus during these interviews was on specialist decision-making in the period directly following the resolution of the initial event, and so patient's opinion and risk factors relating to the period following the discontinuation of treatment (eg, D-dimer levels) were not taken into account. Our questions were informed by a review of the pertinent literature and guidelines, ${ }^{11} 12$ which had yielded an initial selection of relevant attributes. Importantly, we decided to exclude cancer as a risk factor, as its impact on the decision to continue or stop anticoagulation is too dominant: presence of an active cancer invariably warrants indefinite anticoagulation, regardless of other factors. ${ }^{11}$

In our interviews, we specifically asked our interviewers to identify patient characteristics that significantly impact either the risk of VTE recurrence or the risk of bleeding. Although all physicians weighted individual risk factors differently, common factors and their corresponding levels were identified. table 1 is a frequency table of the risk factors that were considered to significantly impact either on VTE recurrence risk or on bleeding risk, by number of physicians who mentioned them. This table does not include risk factors that were mentioned by fewer than two interviewees.

\section{Reducing design complexity}

The preselection of attributes yielded 18 factors that were indicated to be of importance by at least two physicians. A recommendation for conjoint experiments, which exhibit commonalities with our approach, states to limit the complexity of a design as much as possible, as an overly complex design may confuse respondents and distort the responses to an experiment. ${ }^{15}$ We first discarded factors that were unsuitable for the vignette experiment due to their ungeneralisability: uncontrolled hypertension and physical activity. We then removed the 'unstable or high INR' factor on account of it being specific to vitamin $\mathrm{K}$ antagonists. We further trimmed down the list of factors by discarding the risk factors that only rarely occur in real-life patients with VTE: thrombocytopenia $(<1 \%)^{16}$ and liver disease $(\sim 1.5 \%) .{ }^{17}$ Finally, we combined 'signs of PTS' with the 'type of VTE' factor, so as to prevent inappropriate interactions: post-thrombotic syndrome almost exclusively occurs in tandem with DVT, rather than with PE. ${ }^{18}$ This leaves 12 factors in our reduced model.

\section{Experimental design}

Given 12 factors, of which nine have two levels; two have three levels; and one has six levels, there is a total of $2^{9} 3^{2} 6=27648$ level permutations in the full factorial model. As having respondents evaluate all 27648 possible scenarios would be prohibitively costly, we created an efficient fractional factorial design via Fedorov's 1972 exchange algorithm. ${ }^{19}$ To generate this design, we utilised the $\mathrm{R}$ package 'AlgDesign', ${ }^{20}$ an implementation of the Fedorov algorithm. The Fedorov algorithm randomly selects rows from a full factorial matrix $\mathrm{X}$ to create a new matrix $\xi$, and then exchanges rows from $\xi$ with rows from $X l \xi$ to optimise a specified criterion. In our case, we optimised our reduced design for $D$-efficiency and $G$-efficiency.

The number of vignettes in the reduced design was selected on the basis of the following criteria: 
Table 2 Efficiency criteria of design matrix $\xi$

\begin{tabular}{lll}
\hline $\mathbf{N}_{\xi}$ & $\mathbf{D e}_{\xi}$ & $\mathbf{G e}_{\xi}$ \\
72 & 0.998 & 0.94 \\
\hline
\end{tabular}

i. D-efficiency

ii. G-efficiency

iii. Level balance

iv. Feasibility of the required sample size

\section{D-efficiency}

The D-efficiency, perhaps the most frequently used efficiency criterion in the design of experiments (DoE), ${ }^{21}{ }^{22}$ is a measure of information in a reduced design $\xi$ relative to the full factorial matrix $X$. In the AlgDesign package, the $\mathrm{D}$ criterion for a design $\xi$ is computed as the $k$ th root of its generalised variance,${ }^{20}$ that is, the determinant of the normalised covariance matrix $\sum(\xi)=\left(\xi^{T} \xi / N_{\xi}\right)$ :

$$
D_{\xi}=|\Sigma(\xi)|^{\frac{1}{k}}
$$

Where $\mathrm{k}$ is the number of columns and $N_{\xi}$ the number of rows in $\xi$. The relative D-efficiency $D e$ of $\xi$ with respect to $\mathrm{X}$ is computed by taking the ratio of $\mathrm{D}_{\xi}$ to the $\mathrm{D}$ criterion of the full factorial design:

$$
D e_{\xi}=\frac{D_{\xi}}{D_{X}}
$$

\section{G-efficiency}

Gefficiency is another optimality criterion, which aims to minimise the prediction variance of a fractional factorial design. ${ }^{22}$ In the AlgDesign package, G-efficiency is computed as follows $^{20}$ :

$$
G e_{\xi}=\frac{k}{\max _{x \in X}\left(x^{T} \sum(\xi)^{-1} x\right)}
$$

Here, $x$ denotes a row of $X$. In natural language, this means that the number of columns $k$ is divided by the row in $X$ with the highest precision (the inverse of the covariance matrix is the precision matrix ${ }^{23}$ ) of $\xi$ over $X$.

\section{Level balance}

Level balance is an important attribute of experimental designs, which ensures design orthogonality. ${ }^{24}$ In order to achieve level balance, the number of rows $N_{\xi}$ should be an integer multiple of the number of levels for all factors. This ensures that all levels can be represented an equal number of times per factor in the design matrix, creating a balanced set of vignettes. ${ }^{25}$

\section{Feasibility of the required sample size}

There does not appear to be a uniform standard when it comes to determining an appropriate sample size for experimental designs such as the one described in this protocol. One formula, used in conjoint analyses, dictates a sample size of $n=500 \times c /(a \times t)$, where $c$ signifies the maximum number of levels for any of the factors, $a$ denotes the number of choice alternatives presented at once, and $t$ denotes the number of unique vignettes presented to each respondent. ${ }^{26-28}$ Another recommendation states that all vignettes in a design should be evaluated at least six times. ${ }^{29}$

\section{Number of vignettes in the reduced design}

Based on the above considerations, we selected a design $\xi$ with $N_{\xi}=72$. This number of vignettes yielded a wellrounded balance between our efficiency criteria, as illustrated in figure 1 and table 2. To reduce workload for our respondents, we divided this design into six blocks of 12 vignettes each, using the optBlock function in the 'AlgDesign' R package. After optimisation, we achieved a block design diagonality of 0.88 . Plugging in the values for a single block into our sample size formula returns a required sample size of $n=500 \times \frac{6}{1 \times 12}=250$. As there is considerable overlap between blocks, we assume a sample size of 250 to be sufficient to draw reliable inferences with the design as specified above. Following the 'six-times rule', accounting for twelve vignette evaluations per respondent, yields a required sample $n=\frac{72 \times 6}{12}=36$.

\section{Confirmation of the required sample size via a priori power analysis}

As we intend to analyse the experimental results with mixed-effects models (see Analysis of the results section), it is necessary to validate the sufficiency of the above sample size calculation. ${ }^{30}$ We performed a series of simulations, using the $\mathrm{R}$ command 'simulate' and the 'makeLmer' and 'makeGlmer' commands in the $\mathrm{R}$ package 'simr', to construct an a priori power analysis. We conducted two linear mixed effects models and four generalised linear mixed effects models with logit link functions using the $\mathrm{R}$ package 'Ime4' ${ }^{32}$ We used pilot data (see Pilot evaluation of the design section) to construct these models. Specifically, we regressed outcome variables 'VTE recurrence risk' and 'bleeding risk' (see Outcome variables section) on a vector of covariates, consisting of the selected patient risk factors and participating specialist characteristics (see Physician characteristics section). We then systematically reduced these models via AIC-based forward and backward stepwise variable selection, and by eliminating variables with clinically insignificant effect sizes $(<|0.01|$ on a scale $0-10)$ or particularly unpromising $\mathrm{p}$ values $(>0.6)$, as determined via Type II Wald $X^{2}$ tests. We created four binomial generalised linear mixed models to separately analyse the four treatment outcomes in our original pilot survey (continue 3 months; continue 6-12 months; continue indefinitely; cease treatment or switch to aspirin). For the 'continue' treatment options we adapted and modified the 'recurrence risk' model's equation until it exhibited adequate fit and model convergence. For the 'stop treatment' option we used the same process, this time modifying the 'bleeding risk' model's equation. Random slopes and intercepts were included as much as possible; the final selection of mixed effects was based on model improvement and convergence. The recurrence risk model includes 10 covariates, one random 
Table 3 Simulated a priori power analysis: power by sample size

\begin{tabular}{|c|c|c|c|c|c|c|c|c|c|}
\hline & Powe & & & & & & & & \\
\hline Model & $n=10$ & $n=20$ & $n=30$ & $n=40$ & $n=50$ & $n=60$ & $n=100$ & $n=200$ & $n=250$ \\
\hline $\begin{array}{l}\text { Recurrence risk } \\
\text { (LMER) }\end{array}$ & 0.50 & 0.70 & 0.70 & 0.80 & 0.80 & 0.90 & 0.90 & 0.90 & 0.90 \\
\hline $\begin{array}{l}\text { Bleeding risk } \\
\text { (LMER) }\end{array}$ & 0.71 & 0.86 & 0.86 & 0.86 & 0.86 & 1.00 & 1.00 & 1.00 & 1.00 \\
\hline $\begin{array}{l}\text { Treatment } 1 \\
\text { (GLMER) }\end{array}$ & 0.00 & 0.13 & 0.38 & 0.50 & 0.75 & 0.75 & 0.88 & 1.00 & 1.00 \\
\hline $\begin{array}{l}\text { Treatment } 2 \\
\text { (GLMER) }\end{array}$ & 0.00 & 0.00 & 0.20 & 0.20 & 0.60 & 0.40 & 1.00 & 1.00 & 1.00 \\
\hline $\begin{array}{l}\text { Treatment } 3 \\
\text { (GLMER) }\end{array}$ & 0.00 & 0.50 & 0.50 & 0.60 & 0.90 & 0.90 & 0.90 & 1.00 & 1.00 \\
\hline $\begin{array}{l}\text { Treatment } 4 \\
\text { (GLMER) }\end{array}$ & 0.17 & 0.50 & 0.67 & 1.00 & 1.00 & 1.00 & 1.00 & 1.00 & 1.00 \\
\hline
\end{tabular}

slope and three random intercepts. The bleeding risk model features 7 covariates, two random slopes and three random intercepts. The model corresponding to treatment 1 features 8 covariates and a random intercept for participants. Logistic model 2 includes 5 covariates and three random intercepts (participant, block identifier, country). Logistic model 3 incorporates 7 covariates and random intercepts for participant and block identifier. Logistic model 4 features 6 covariates and three random intercepts (participant, block identifier, country).

From these models created on the basis of pilot data, we extracted the covariate coefficients $(\widehat{\beta})$, the variance and correlation components and the residual SD $(\widehat{\sigma})$. We then generated covariate $(X)$ matrices corresponding to varying sample sizes by concatenating blocks of vignettes and simulating plausible participant characteristics. Finally, we simulated responses for each of the models at variable sample sizes. We defined the power of all models as the proportion of incorporated covariates with $\mathrm{p}<0.05$, assessed with Type II Wald $X^{2}$ tests. The power analysis is summarised in table 3 and figure 2. The power analyses indicate that the lower bound of the sample size should be adjusted to $n=100$. The original upper bound of $n=250$ was validated as amply sufficient to estimate coefficients with reliable power.

\section{Pilot evaluation of the design}

In May 2016, we sent out Microsoft Word-based pilot surveys to 12 thrombosis experts to evaluate our initial design. From the comments we received, it was apparent that some of the factors and levels in our model were not formulated precisely enough. For instance, pilot participants indicated that in patients who had had a previous VTE, it was important to know when this had occurred.

\section{Power by sample size}

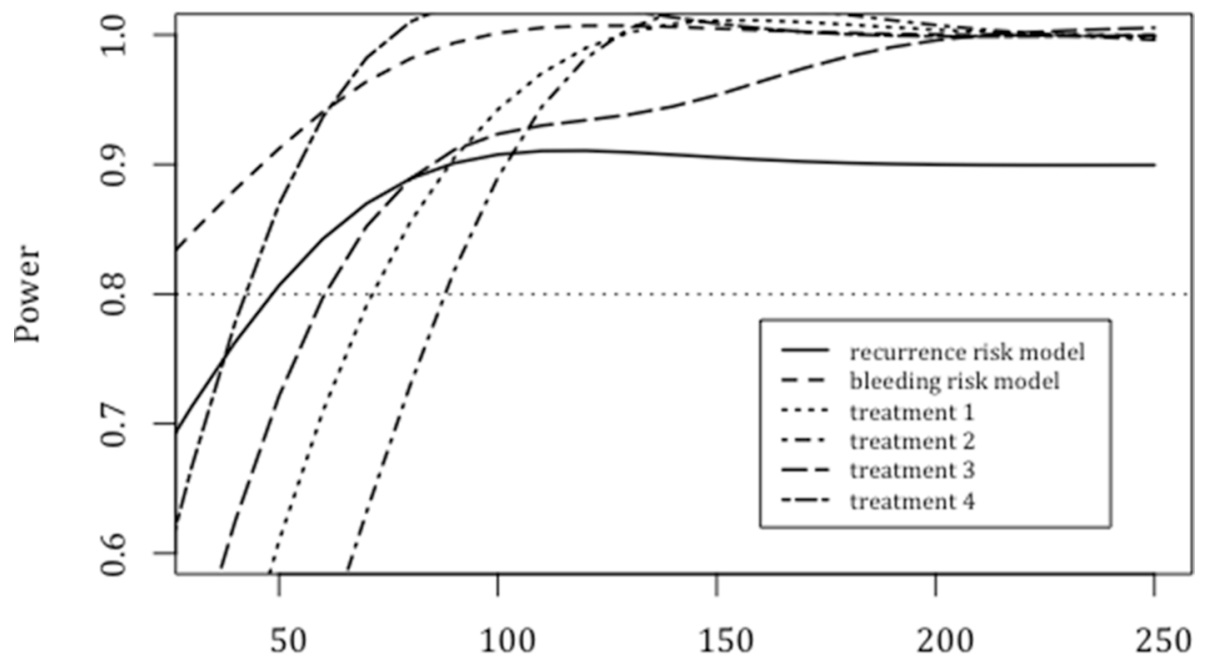

Figure 2 Power by sample size. 


\begin{tabular}{|c|c|c|c|c|c|c|}
\hline Factor & Level 1 & Level 2 & Level 3 & Level 4 & Level 5 & Level 6 \\
\hline Sex & female & male & & & & \\
\hline Age & $<65$ & $>65$ & & & & \\
\hline BMI & underweight & normal weight & overweight & & & \\
\hline Idiopathic & no & yes & & & & \\
\hline Type of VTE & distal DVT & $\begin{array}{l}\text { distal DVT with } \\
\text { signs of PTS }\end{array}$ & proximal DVT & $\begin{array}{l}\text { proximal DVT } \\
\text { with signs of } \\
\text { PTS }\end{array}$ & $\begin{array}{l}\text { non- } \\
\text { massive PE }\end{array}$ & massive $\mathrm{PE}$ \\
\hline Previous VTE & no & $\begin{array}{l}\text { yes ( } 2 \text { years } \\
\text { ago) }\end{array}$ & & & & \\
\hline Family history of VTE & no & yes & & & & \\
\hline History of major bleeding & no & yes & & & & \\
\hline Thrombophilia & none & acquired & inherited & & & \\
\hline Renal function & normal & $\begin{array}{l}\text { insufficient } \\
(<50 \mathrm{~mL} / \mathrm{min})\end{array}$ & & & & \\
\hline Alcohol or substance abuse & no & yes & & & & \\
\hline Absolute indication for aspirin & no & yes & & & & \\
\hline
\end{tabular}

BMI, body mass index; DVT, deep vein thrombosis; PE, pulmonary embolism; VTE, venous thromboembolism.

For this item, we added a timeframe to improve clarity. Some participants struggled with the unqualified formulation 'renal function: insufficient'. We included the more detailed description ' $<50 \mathrm{~mL} / \mathrm{min}$ ' in these cases, referring to the corresponding creatinine clearance rate. Additionally, of patients who received concurrent antiplatelet therapy, a number of pilot participants said to be confused as to whether the practitioner treating the patient for VTE recurrence could cease this concurrent therapy. As our intention was to refer to patients with an absolute indication for antiplatelet therapy, we reformulated this item to more explicitly refer to such patients. With these changes, we arrived at our final design, shown in table 4.

\section{Physician characteristics}

From our expert interviews, it was apparent that physicians involved in the secondary prevention of VTE all have unique perspectives on thrombosis and bleeding risk in VTE. In order to understand why physicians who are faced with the same clinical vignettes may respond to these differently, we decided to include a number of questions relating to characteristics of the physicians themselves. Before commencing the experiment, physicians are asked to provide their age, gender, country of employment, specialty, years of experience with the treatment of VTE, and an estimate of the number of unique patients with VTE they treat per annum. These physician characteristics were selected on the basis of previous research into physician guideline adherence and clinical decision-making. ${ }^{33-37}$

By incorporating the 'country of employment' variable as a random effect, we hope to capture the variance resulting from any country-specific aspects of physician's decision-making that would otherwise be difficult to quantify.
We provided six possible answers to choose from in response to the specialty question, that is,: internal medicine, vascular medicine/angiology, cardiology, haematology, pulmonology or other. The specialty of responding physicians will also be included as a random effect in our analysis.

\section{Outcome variables}

As outcome variables, we incorporated two continuous variables to respectively estimate VTE recurrence risk and bleeding risk on a 100-points visual analogue scale indicating low to high risk, and five discrete variables: continue treatment for three additional months, continue 9 additional months, continue indefinitely, stop treatment, or switch to aspirin. These choices were based on the most recent CHEST and ESC VTE treatment guidelines, ${ }^{11}{ }^{12}$ the conducted expert interviews and the comments received on our pilot design.

\section{Implementation of the survey}

The survey has been implemented online by independent web developers and is currently live at www.vte-survey.com (figure 3). Survey respondents are randomised to one of six pre-specified blocks at the start of the experiment.

As mentioned above, one aspect of our experimental design was to make the workload for study participants as slight as possible. For this reason, in the online implementation of the survey the progress of participants is continuously recorded. This allows participants to take breaks during the survey and even to leave the website, without their responses up until that point being forfeited. Participants may return to the website at any time and resume the survey where they left off. In our current sample of respondents, there is a small number of such cases that skews the mean survey completion time 


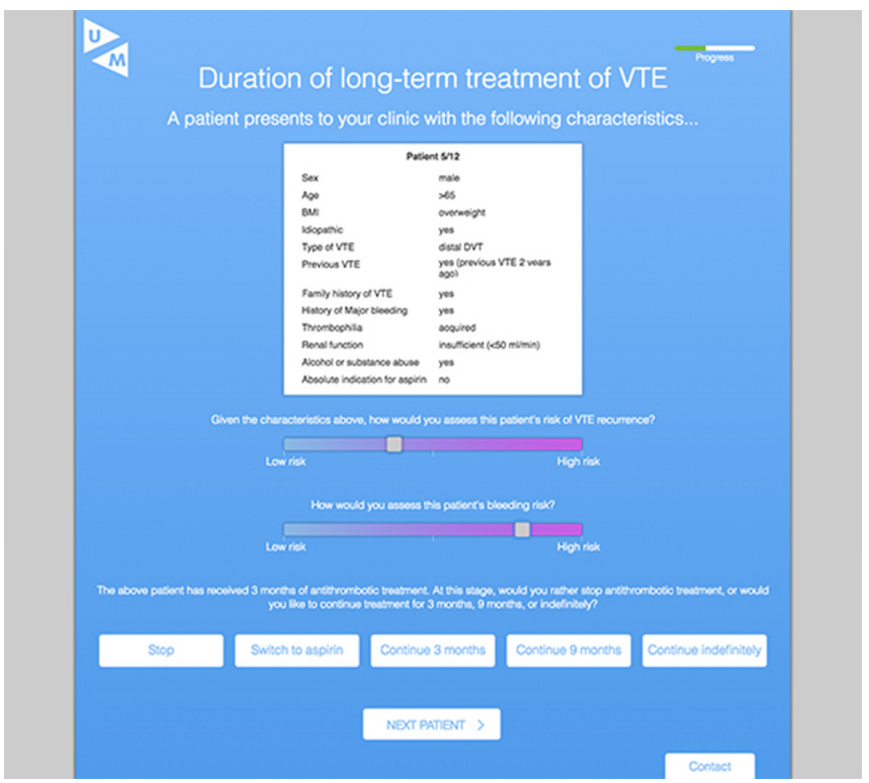

Figure 3 Screen capture of the web-based survey (www. vte-survey.com).

considerably upward. A better reflection of the average workload is given by the median survey completion time, which in our current sample is equal to $9 \mathrm{~min}$. Based on this number, we conclude that the risk of participant fatigue is negligible for our study.

Data collection was commenced in July 2016, and is still ongoing. We have reached our stated sample size requirements in January 2017, but will leave the website live for at least the remainder of this year, so that more data may be recorded for future analysis. We welcome any specialists involved in the preventive treatment of VTE to participate in this experiment.

\section{Participant recruitment strategy}

As we are interested in specialist decision-making, our recruitment strategy focuses exclusively on specialists. This group of subjects is particularly difficult to reach in a non-systematic or stochastic manner. This section details how we approached the recruitment of participants in a way that we believe maximised the chance of reaching a broad and representative sample.

In order to meet the sample size requirements, as determined by the vignette-based sample size formulae as well as the power analysis described above, we have leveraged several channels to recruit study participants so far. First, we contacted a list of several hundred specialists who participate or have participated in the Einstein Choice study. ${ }^{14}$ Participation in this context is defined as having recruited at least one patient for the Einstein Choice study, and the list features specialists of variable ages, professional stature and levels of experience. We also contacted professional collectives of specialists, such as the International Society of Thrombosis and Haemostasis (ISTH), the ESC and the Asian Pacific Society on Thrombosis and Hemostasis (APSTH), which led to calls for participation in the ISTH (October 2016) and
APSTH newsletters (January 2017) and on their respective websites, and a call for participation on the youth community (EAPC) of the ESC on LinkedIn. Finally, we asked each specialist who responded favourably to our request to participate to disseminate the survey among colleagues and residents.

\section{Analysis of the results}

We will conduct several separate analyses of our results (figure 4). A first linear mixed effects regression model will relate all risk factors and physician characteristics to the continuous estimated VTE recurrence risk outcome variable. A second model does the same for bleeding risk. After having estimated the coefficients in these two linear models, we will use generalised linear mixed models (GLMMs) to estimate the ORs for all independent variables, including physician characteristics, in relation to the choice of treatment options. We will use a GLMM with a binomial logit link function for grouped outcomes (stop vs continue treatment), and a multinomial logit regression to discover which variables best predict the five separate treatment outcomes.

In the linear models, we will evaluate model fit via diagnostic plots (AV plots, Q-Q plots, standardised residuals vs fitted plots), the adjusted $R^{2}$ and relative measures such as the Akaike Information Criterion (AIC). In the logistic models, we will employ diagnostic plots, the area under the receiver operating characteristic curve (AUROC), McFadden's pseudo- $R^{2}\left(\rho^{2}\right)^{38}$ and the predictive accuracy of the models on a test set (kept separate from the development set) to evaluate model fit.

The choice for mixed effects analyses was informed by the theoretical assumption that there is considerable inter-physician variability, and to control for random variance associated with aspects of no interest (eg, block allocation). While analysis of the fixed effects (ie, the regression coefficients) will provide general insights as to which features are of particular interest in determining a patient's risk of VTE recurrence or bleeding, it is the decomposition of the error term that will show us specifically where and to what extent physicians disagree with one another. To this end, we will specify random slopes that vary by physician for as many relevant features as possible. Our intention in this regard is to keep random effects structures 'maximal', although within the realistic constraints imposed by the data. ${ }^{30}$ All analyses will be conducted in the statistical software package R.

\section{IMPLICATIONS OF THIS RESEARCH}

The research described in this protocol will provide insight into the mechanisms underlying the decision-making of physicians involved in the outpatient treatment of VTE. Our aims are to identify the relative weights of risk factors; to determine how physicians decide on the duration of preventive treatment, and to uncover what prompts physicians to discontinue further treatment. 


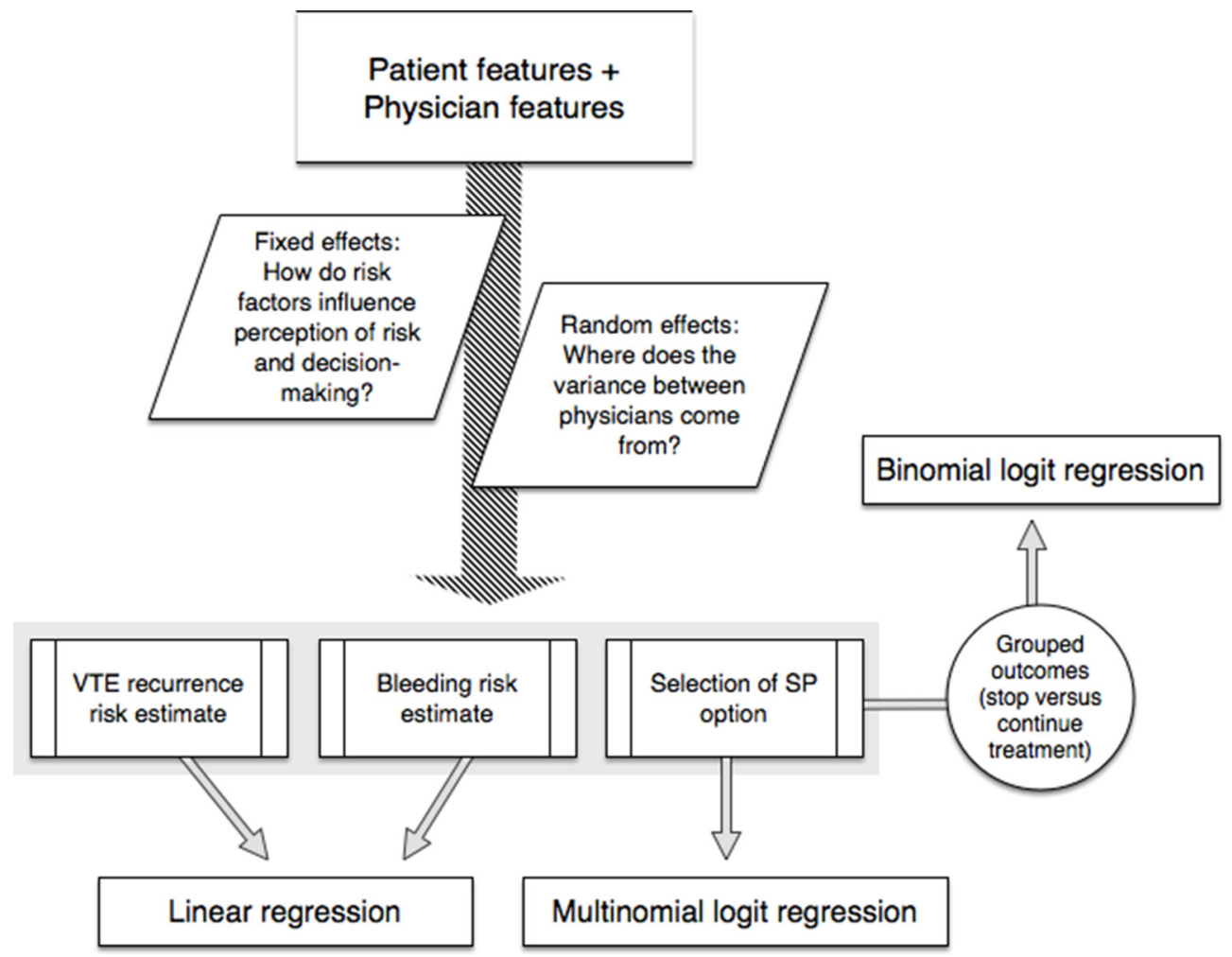

Figure 4 Analysis flowchart.

Simultaneously, this research places a strong focus on the variance that exists between physicians in determining VTE treatment duration. We will explore which factors contribute to this variance by using a mixed-effects approach, enabling direct analysis of the inter-individual differences in decision-making patterns. In so doing, it is our hope that this research will lead to a better understanding of why physicians may deviate from the best-practice guidelines, and what biases may potentially be at play in making this decision.

We believe that the insights acquired through this study will benefit physicians involved in the treatment of VTE, by elucidating the overlaps and discrepancies between treatment strategies and by highlighting the areas potentially prone to oversights. For this same reason, our findings should also be of benefit to the national and international collectives whose aim is to unify and coordinate VTE treatment. By explicitly showing where physician decision-making coincides and where it varies, we believe that VTE prevention education and policy-making can at once be made more efficient and more effective.

\section{ETHICS AND DISSEMINATION}

All data are de-identified, and any identifying characteristics of the respondents will not be reported in a final manuscript or elsewhere. This study does not make use of real patients, and participant data are not released to third parties. For these reasons, this study is exempt from the requirement to gain approval from a medical research ethics committee (MREC).
A paper containing a qualitative summary of the expert interviews is currently under peer review. We are currently working on a manuscript that contains the analysis of the results of the experiment described in this protocol. When completed, this article will also be submitted to a peer-reviewed journal.

Acknowledgements We extend our gratitude to the thirteen interviewees whose input helped us select risk factors and outcome variables for our vignette experiment. Our thanks also go out to Maastricht University Medical Center's thrombosis department, Dr Avi Leader of Israel and Dr Michael Nagler of Switzerland for their valuable comments and evaluation of our pilot survey. We further thank Petro van Bergen for his help in recruiting survey participants, the ISTH and the APSTH for featuring this experiment in their newsletters (October 2016; January 2017). We thank the youth community of the ESC for featuring a call for participation on their youth community's Linkedln page. We are grateful to D David McAneny of Boston University, Dr Charles Mahan of the University of New Mexico and Dr José A. López-López of the University of Bristol, whose comments have inspired substantial revision of this manuscript. Lastly, we are indebted to all survey participants for contributing to our research.

Contributors VTC and MHP designed the protocol. VTC wrote the protocol. BABE provided helpful input throughout.

Competing interests VTC and BABE declare no competing interests. MHP has received research support and honoraria, and participated in advisory boards for Bayer HealthCare, Sanofi-Aventis, Boehringer Ingelheim, GlaxoSmithKline, Daiichi Sankyo, LeoPharma, ThromboGenics, and Pfizer.

Provenance and peer review Not commissioned; externally peer reviewed.

Open Access This is an Open Access article distributed in accordance with the Creative Commons Attribution Non Commercial (CC BY-NC 4.0) license, which permits others to distribute, remix, adapt, build upon this work non-commercially, and license their derivative works on different terms, provided the original work is properly cited and the use is non-commercial. See: http://creativecommons.org/ licenses/by-nc/4.0/ 
(C) Article author(s) (or their employer(s) unless otherwise stated in the text of the article) 2017. All rights reserved. No commercial use is permitted unless otherwise expressly granted.

\section{REFERENCES}

1. Cushman M. Epidemiology and risk factors for venous thrombosis. Semin Hematol 2007;44:62-9.

2. White $\mathrm{RH}$. The epidemiology of venous thromboembolism. Circulation 2003;107:41-8.

3. Baser O. Prevalence and economic burden of venous thromboembolism after total hip arthroplasty or total knee arthroplasty. Am J Manag Care 2011;17:S6-S8.

4. Zhu T, Martinez I, Emmerich J, et al. Treatment, and public awareness. Arterioscl Throm Vas 2009;29:298-310.

5. Beyer-Westendorf J, Cohen AT, Monreal M. Venous thromboembolism prevention and treatment: expanding the rivaroxaban knowledge base with real-life data. Eur Heart J Supp 2015;17:D32-D41.

6. Sternitzky R, Hochauf S, Schellong SM. [Secondary prophylaxis of venous thromboembolism]. Hamostaseologie 2007;27:32-40.

7. Hibbert PD, Hannaford NA, Hooper TD, et al. Assessing the appropriateness of prevention and management of venous thromboembolism in Australia: a cross-sectional study. BMJ Open 2016;6:e008618.

8. Caprini JA, Tapson VF, Hyers TM, et al; NABOR Steering Committee. Treatment of venous thromboembolism: adherence to guidelines and impact of physician knowledge, attitudes, and beliefs. J Vasc Surg 2005;42:726-33.

9. Brenner B. Venous thromboembolism: advances and controversies. Best Pract Res Clin Haematol 2012;25:233.

10. Schulman S, Douketis J. Secondary prevention of venous thromboembolism. Bmi 2013;347:f5440.

11. Kearon C, Akl EA, Ornelas J, et al. Antithrombotic therapy for VTE disease: chest guideline and expert panel report. Chest 2016;149:315-52.

12. Konstantinides $\mathrm{S}$, et al. ESC guidelines on the diagnosis and management of acute pulmonary embolism. Eur Heart $J$ 2014;2014:3033-80.

13. Evans SC, Roberts MC, Keeley JW, et al. Vignette methodologies for studying clinicians' decision-making: validity, utility, and application in ICD-11 field studies. Int J Clin HIth Psyc 2015;15:160-70.

14. Bayer. Reduced-dosed Rivaroxaban in the Long-term Prevention of Recurrent Symptomatic VTE (Venous Thromboembolism) (Einstein Choice). In: Clinical trials gov [Internet]. Bethesda (MD): National Library of Medicine (US), 2000. https://clinicaltrials.gov/ct2/show/ NCT02064439 NLM Identifier: NCT02064439 (cited 2016 Oct 27).

15. DeShazo JR, Fermo G. Designing choice sets for stated preference methods: the effects of complexity on choice consistency. J Environ Econ Manage 2002;44:123-43.

16. Stein PD, Hull RD, Matta F, et al. Incidence of thrombocytopenia in hospitalized patients with venous thromboembolism. Am J Med 2009;122:919-30.

17. Saleh T, Matta F, Alali F, et al. Venous thromboembolism with chronic liver disease. Am J Med 2011;124:64-8.

18. Bova C, Rossi V, Ricchio R, et al. Incidence of post-thrombotic syndrome in patients with previous pulmonary embolism. A retrospective cohort study. Thromb Haemost 2004;92:993-6.
19. Fedorov VV. Theory of optimal experiments. Elsevier 1972.

20. Wheeler RE. Comments on algorithmic design 2004-2009. http:// cran.r-project.org/web/packages/AlgDesign/vignettes/AlgDesign.pdf. (accessed April 2016)

21. Kuhfeld WF. Efficient experimental designs using computerized searches: Research Paper Series, SAS Institute, Inc, 1997.

22. Rodríguez M, Jones $B$, Borror $\mathrm{CM}$, et al. Generating and assessing exact G-optimal designs. Journal of quality technology 2010;42:3.

23. Wasserman L. All of statistics. A concise course in statistical inference: Springer Science \& Business Media, 2013.

24. Collins LM, Dziak JJ, Li R. Design of experiments with multiple independent variables: a resource management perspective on complete and reduced factorial designs. Psychol Methods 2009;14:202-24.

25. Kuhfeld WF, Tobias RD, Garratt M. Efficient experimental design with marketing research applications. J Mark Res 1994;31:545-57.

26. Rose JM, Bliemer MCJ. Sample size requirements for stated choice experiments. Transportation 2013;40:1021-41.

27. Vilikus O. Optimization of sample size and number of tasks per respondent in conjoint studies using simulated datasets. Studia Ekonomiczne/Uniwersytet Ekonomiczny w Katowicach, 2012:77-83.

28. Engel J, van der Wulp I, Poldervaart JM, et al. Clinical decisionmaking of cardiologists regarding admission and treatment of patients with suspected unstable angina or non-ST-elevation myocardial infarction: protocol of a clinical vignette study. BMJ Open 2015;5:e006441.

29. Louviere JJ, Hensher DA, Swait JD, et al. Analysis and application. New York: Cambridge University Press, 2000:83-111.

30. Matuschek H, Kliegl R, Vasishth S, et al. Balancing type I error and power in linear mixed models. J Mem Lang 2017;94:305-15.

31. Green P, MacLeod CJ. SIMR: an R package for power analysis of generalized linear mixed models by simulation. Methods Ecol Evol 2016;7:493-8.

32. Bates D, Mächler M, Bolker B, et al. Fitting linear Mixed-Effects models using Ime4. J Stat Softw 2015;67:1-48.

33. Choudhry NK, Fletcher RH, Soumerai SB. Systematic review: the relationship between clinical experience and quality of health care. Ann Intern Med 2005;142:260-73.

34. Solomon DH, Brookhart MA, Gandhi TK, et al. Adherence with osteoporosis practice guidelines: a multilevel analysis of patient, physician, and practice setting characteristics. Am J Med 2004;117:919-24.

35. Kumar A, Fonarow GC, Eagle KA, et al; REACH Investigators. Regional and practice variation in adherence to guideline recommendations for secondary and primary prevention among outpatients with atherothrombosis or risk factors in the united states: a report from the REACH registry. Crit Pathw Cardiol 2009;8:104-11.

36. McKinlay JB, Lin T, Freund K, et al. The unexpected influence of physician attributes on clinical decisions: results of an experiment. $J$ Health Soc Behav 2002;43:92-106.

37. Berthold HK, Gouni-Berthold I, Bestehorn KP, et al. Physician gender is associated with the quality of type 2 diabetes care. J Intern Med 2008;264:340-50.

38. McFadden D. Conditional logit analysis of qualitative choice behavior, 1973.

39. Barr DJ, Levy R, Scheepers C, et al. Random effects structure for confirmatory hypothesis testing: keep it maximal. J Mem Lang 2013;68:255-78. 